\title{
High-Resolution Image Sharing (HIS) on WhatsApp: A Method to Facilitate Instant and High-Quality Case Consultations in Cytopathology
}

WhatsApp (WhatsApp ${ }^{\oplus}$ Inc., Mountain View, California, USA) is one of the most widely used free smartphone applications allowing two or more users to exchange messages, images, audio or video easily and quickly via an Internet connection (1). It has been used efficiently among pathologists to obtain a fast second opinion, share interesting aspects of daily cases, and increase the awareness and interest of students in pathology (2-5). This platform also serves as an alternative to the microscope-mounted digital cameras particularly for the ones in undeveloped countries devoid of these devices (6). Cytopathologists and those interested in cytopathology (CPs) have been increasingly using WhatsApp to take advantage of these benefits and more.

WhatsApp users typically send photographs as "image files" either from their Gallery file or by tapping the camera icon in the recipient's profile. This conventional way of sharing naturally decreases the quality of images after delivery to save storage memory. However, low-quality images from cytopathology cases yield blurred cellular details when zoomed in, which is not convenient for a precise cytopathologic evaluation.

In this article, an image sharing technique called "highresolution image sharing (HIS)" is described where the quality of images is maintained and zooming in does not significantly impair the cytologic details in contrast with the conventional way.

A compound light microscope, Carl Zeiss Axio Scope A1 (Carl Zeiss Microimaging GmbH, Göttingen, Germany), was used to obtain the microphotographs. The eyepiece and objective lenses of the microscope were cleaned using a lens tissue. A few drops of xylene were used to remove balsam from the objective lenses. The light was centered and the substage condenser was focused.

The slides from three different cases were obtained from the pathology slide archive. Each slide was selected for its distinctive cytologic features. The area selected for photography was an area with multiple prominent nucleoli in Case 1 with a diagnosis of reactive mesothelial cells; an area with intranuclear cytoplasmic pseudoinclusion in Case 2 with a diagnosis of papillary thyroid carcinoma; and an area with dysplastic cells in Case 3 with a diagnosis of low-grade squamous intraepithelial lesion.
All the slides were cleaned and then placed on the stage of the microscope. The photographs are taken manually using a Samsung Galaxy S6 smartphone (Samsung Electronics Co. Ltd., South Korea) through one eyepiece. In the camera settings, the aspect ratio was set to 16:9 (16 megapixels (MP)) with a $5 \mathrm{~K}$ resolution $(5312 \times 2988)$. The rear camera was first cleaned of dust with a piece of tissue.

The HIS technique is described for each of the most commonly used mobile operating systems, Android and iOS (Figures 1A-F-2A-J). At the time this article was written, WhatsApp versions for Android and iOS devices were 2.18.380, updated on 11 December, 2018, and 2.18.111, updated on 14 December, 2018, respectively.

Each photograph was sent via the conventional and HIS methods to a recipient on WhatsApp. The areas of interest were equally zoomed in on the matching images until seeing the cellular details, and then screenshot images were taken for comparison of the two methods.

The initial sizes of the photographs were 1.8 megabytes (MB), 4.79 MB, and 1.38 MB in the of Case 1, Case 2, and Case 3, respectively. After sending them via the conventional way, the sizes of the images decreased to 64 kilobytes (KB), $174.64 \mathrm{~KB}$, and $41.55 \mathrm{~KB}$, respectively. The resolutions decreased from $5312 \times 2988$ to $747 \times 1328$ for all images. However, the sizes and resolutions of the images were fully maintained in all images after sending via the HIS technique.

Zooming-in on the shared images on WhatsApp revealed that the cytological details were excellent on X200 and X400 magnifications and of much greater quality on X100 magnification using the HIS technique compared to the conventional method (Figure 3A-F).

The HIS technique proves that it is possible to share an image on WhatsApp without compromising its original size and resolution. Therefore, this technique can be easily adapted to the cytopathologic consultations on WhatsApp.

Cellular details are of the utmost importance for a definite cytopathologic evaluation. Therefore, it is necessary to send high-resolution (HR) images to the recipient $\mathrm{CP}$ during a consultation process on WhatsApp. The decrease in the quality of images is one of the most important issues deterring CPs from WhatsApp case consultations. For 
example, in a recent study, it was stated that both nuclear and cytoplasmic details cannot be seen in the images using X100 or X200 magnifications. For this reason, 400X magnification was used in $91 \%$ of 172 images and 200X magnification was used in the remaining ones for conducting this study (6). It was also documented that there was a discordance in $15.69 \%$ of cases between microscopic diagnoses and smartphone diagnoses. The insufficient quality of WhatsApp images was one of the important reasons reported for the diagnostic discrepancies (6).

There are basically three steps to provide HR images to the recipient $\mathrm{CP}$ on WhatsApp. The first step is to have a smartphone that is capable of taking HR photographs. Currently available smartphones on the market have more than adequate specifications to ensure interpretable microimages with more than $10 \mathrm{MP}, \mathrm{X} 10$ zoom, and $1000 \times 1000$ resolution with a fast autofocus feature. For obtaining the highest quality images, it is suggested to use one of the latest smartphone models and make sure of setting the highest standards in the camera options in advance. In this article, the microimages were taken using a smartphone model released about three years ago. Using a recent smartphone model with higher standard camera features would provide even greater image quality. Second, maintenance of the light microscope should be carried out. The user should clean the eyepiece and objective lenses, center the light, and focus the substage condenser (7). The third step is to take photographs through one eyepiece,
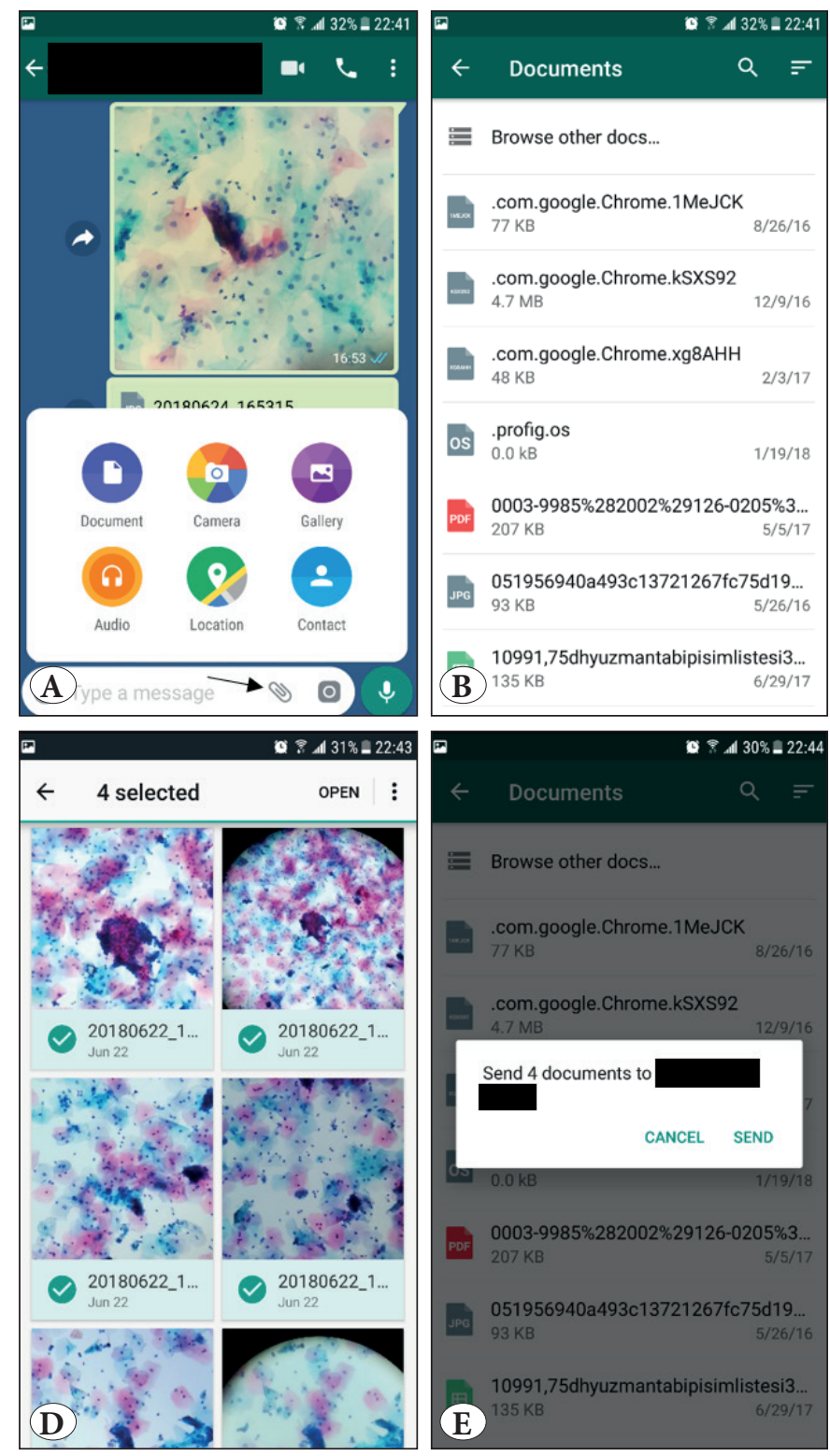
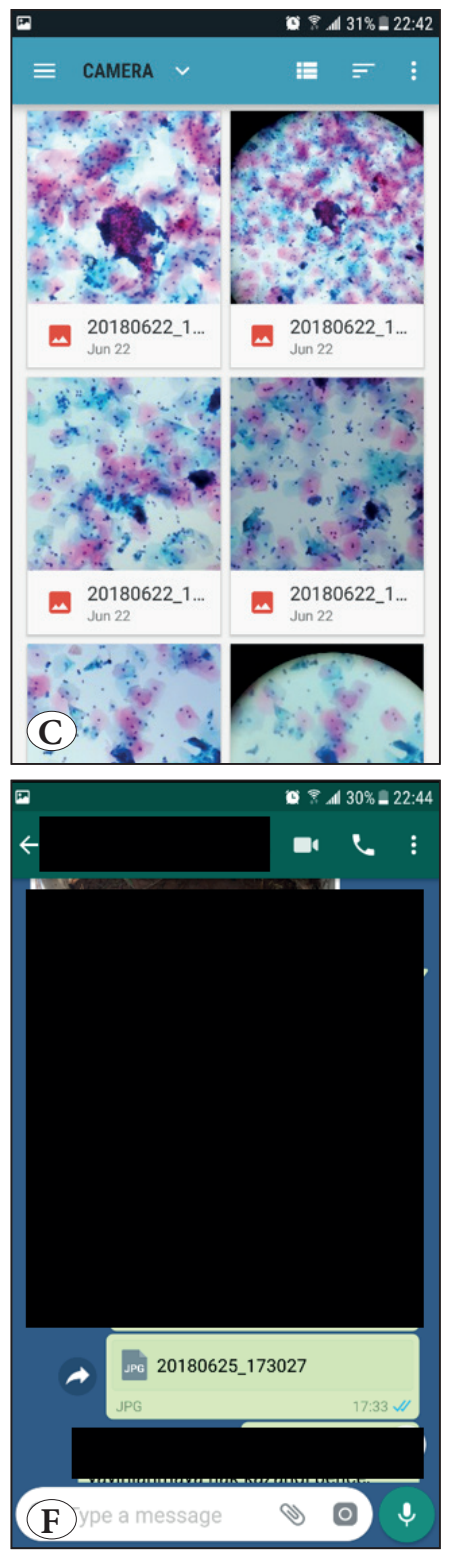

Figure 1: Step by step explanation of how to send images without compromising the quality for Android users. A) Open the recipient's profile, tap the paper clip icon (arrow) and select "Document". B) Select "Browse other docs...”. C) Select the images you want to share. To browse all image files, touch the option on the upper left corner D) For multiple image selection, hold your touch on one of the images, then tap on the other images one by one. After that, tap "Open" on the upper right corner.

E) Send the images.

F) The image(s) are sent. (A Samsung Galaxy S6 smartphone on Android version 7.0 was used) 
whether manually or using a microscope adaptor designed for smartphones. Fourth, an internet connection must be enabled to upload and send the photographs via WhatsApp. The last step is the sharing method on WhatsApp by which the original quality of the shared images must be preserved. There are a few ways to upload and send microphotographs on WhatsApp without changing their original quality (8). The simplest and most effective way suggested in this article is called HIS and enables sending images as "document files" instead of image files. Sending a document is only available for users on iOS 8 and later but there is no such specification listed for Android users. The major drawback of this technique could be that the shared images occupy more memory space than the conventional way since these images are of higher quality. It is suggested to back up and delete the HR images after a period of time if there is a shortage of storage memory.
Another insignificant downside is a slight increase in file transfer time. The transfer time of the images depend on the total document size, the connection type (e.g., modem, ADSL, LAN), and the download speed. For example, a $4 \mathrm{MB}$ image using an ADSL with 8 megabits per second download speed would take about 4 seconds to download. Online download time calculators can be used to estimate the time that will take to transfer images. Although the maximum allowed number of images as document files is not specified on the WhatsApp website, currently up to $100 \mathrm{MB}$ file size is allowed at one time. Several other highquality image sharing methods, albeit much less practical, are also described on this website: https://www.techbii. com/send-full-resolution-whatsapp-image/.

High-resolution image sharing would bring CPs to the next level in WhatsApp consultations. In this author's daily practice, this technique has been tested and used highly
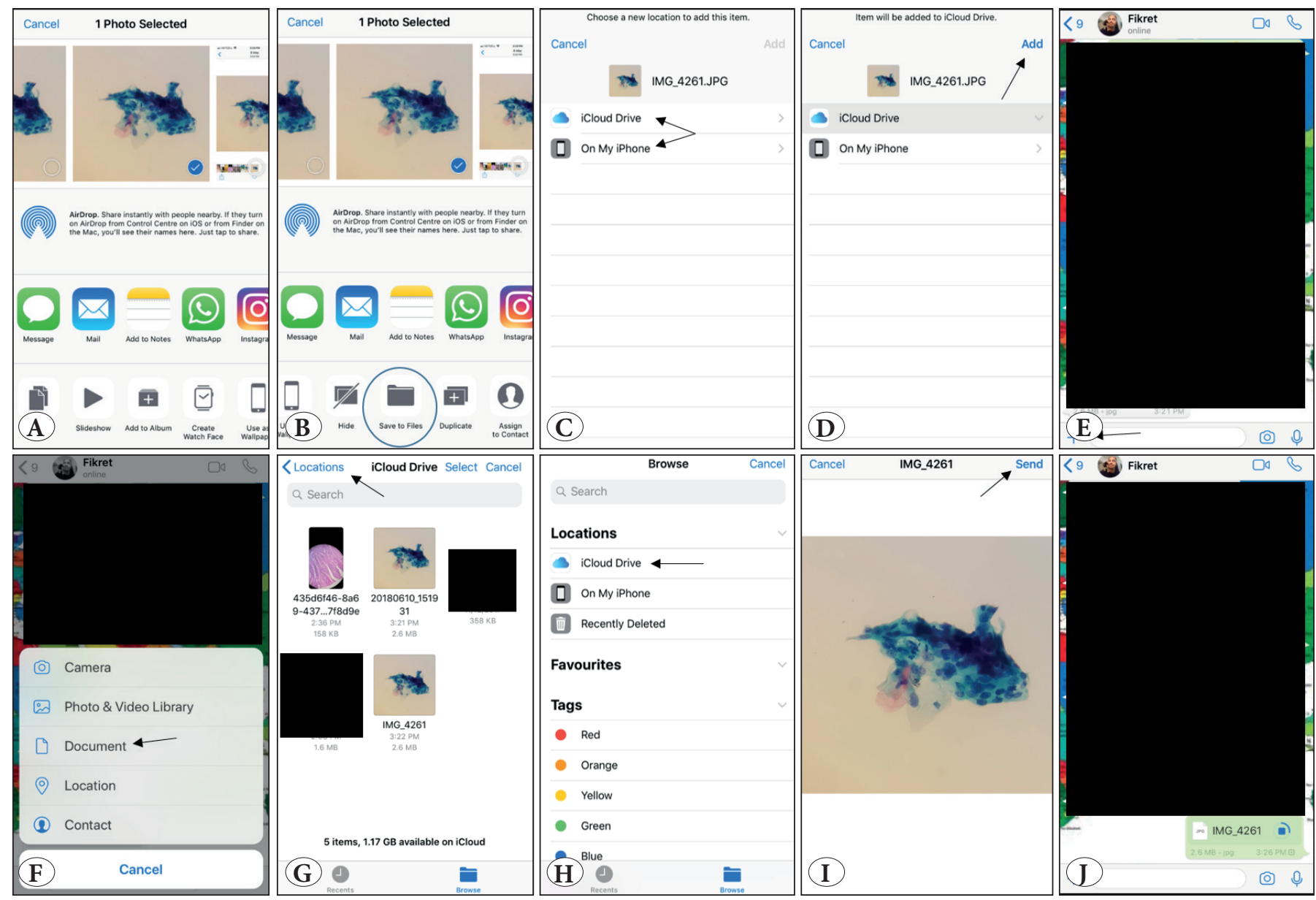

Figure 2: Step by step explanation of how to send images without compromising the quality for iOS users. A) Open Gallery and select the images. B) Select "Save to Files". C) Select one of either file. D) Tap “Add”. E) Open the recipient's profile and tap the Attach (+) icon. F) Select "Document" file. G) Tap "Locations" if the image(s) are not seen in the default file. H) Select the file in which you saved your images. I) Select the images and tap "Send". For multiple image selection, tap "Select" on top of the screen and then tap on the images one by one. J) The image(s) are sent. (An iPhone 6S smartphone on iOS version 12.1.2 was used) 
efficiently. It is believed that this method will be useful not only in cytopathology but also in other fields in pathology and medicine where using HIS would be beneficial $(6,9)$. In future studies on telecytologic consultations on WhatsApp, it is strongly suggested that images be sent using the HIS technique to reduce the risk of interpretation errors compared to microscopic examination pointed in the literature $(3,6)$.

\section{ACKNOWLEDGEMENT}

I would like to thank Ms. Beyza Bircan, who is a student at the Department of Psychology, Eastern Mediterranean University, for her kind help in describing the technique for iOS users.
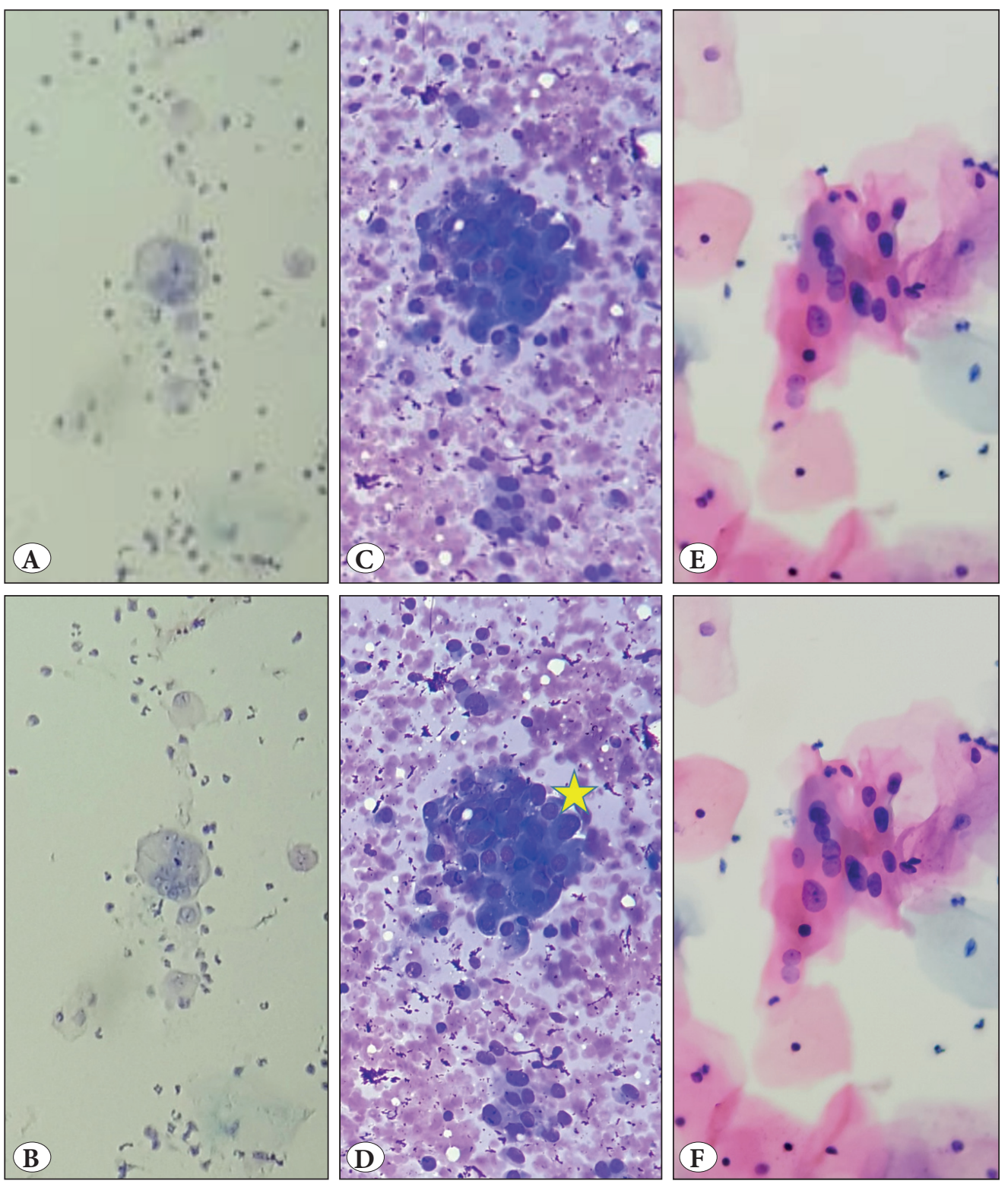

Figure 3: These images display zoomed-in areas from the microphotographs taken at X100, X200, and X400 magnification.

A, B) Case 1, reactive mesothelial cells, (Papanicolaou stain; $x 100$ )

C, D) Case 2, papillary thyroid carcinoma, (Giemsa stain; x200). E, F) Case 3, low-grade squamous intraepithelial lesion, stain (Papanicolaou; x400).

When zooming in on the images, the cytologic details are severely blurred (A and C) and relatively preserved (E) using the conventional way. However, sharing with the HIS technique preserves the quality of the images and the nuclear and cytoplasmic details could be easily evaluated (B, D, and F). The yellow star indicates an intranuclear cytoplasmic pseudoinclusion in a papillary thyroid carcinoma which can only be seen in the image shared by the HIS technique (D). 
6. Sahin D, Hacisalihoglu UP, Kirimlioglu SH. Telecytology: Is it possible with smartphone images? Diagn Cytopathol. 2017;46:406.

7. Davidson MW. ZEISS Microscopy Online Campus. Kohler Illumination. http://zeiss-campus.magnet.fsu.edu/articles/ basics/kohler.html (accessed June 30, 2018).

8. Puthiya S. 5 Ways to Send Pictures in WhatsApp without Losing Quality. https://www.techbii.com/send-full-resolutionwhatsapp-image/ (accessed June 28, 2018).

9. Boulos M, Giustini DM, Wheeler S. Instagram and WhatsApp in Health and Healthcare: An Overview. Futur Internet. 2016;8:37.

\section{Fikret DİRİLENOĞLU (D)}

Department of Pathology,

Near East University, Faculty of Medicine,

CYPRUS, 99138

E-mail: fikretdirilenoglu@gmail.com

Phone: +9054285366 65 